\title{
MicroRNA-31 inhibits tumor invasion and metastasis by targeting RhoA in human gastric cancer
}

\author{
FULIN GE, CHANGZHENG WANG, WEIHUA WANG, WENHUI LIU and BENYAN WU \\ Department of Gastroenterology, Division of Southern Building, Chinese PLA General Hospital, \\ Beijing 100853, P.R. China
}

Received July 28, 2016; Accepted May 18, 2017

DOI: $10.3892 / o r .2017 .5758$

\begin{abstract}
Previous studies have shown that microRNA-31 (miR-31) functions as a tumor-suppressor in various types of cancer. In the present study we found that miR-31 was significantly downregulated in gastric cancer (GC) as determined by microRNA (miRNA) array screening analysis. Although aberrant expression of miR-31 has been found in different types of cancer, its pathophysiological effect and role in tumorigenesis still remain to be elucidated. In the present study, we detected miR-31 expression in both metastatic GC cell lines and tissues that are potentially highly metastatic by real-time polymerase chain reaction (PCR). Transwell and scratch healing assays were conducted to examine whether the ectopic expression of miR-31 could modify the invasion and migration abilities of GC cells in vitro. We found that miR-31 inhibited GC metastasis in a nude mouse xenograft model of GC. Luciferase reporter assays demonstrated that miR-31 could directly bind to the 3 ' untranslated region of RhoA and downregulate the expression of RhoA. Significant downregulation of miR-31 in 78 GC tissues and four GC cell lines was examined by real-time reverse transcription-PCR. Moreover, the decreased expression of miR-31 was demonstrated to be associated with lymph node metastasis, poor $\mathrm{pT}$ and $\mathrm{pN}$ stage, and invasion ability into lymphatic vessels as determined by the Mann-Whitney U test. We also found that miR-31 could inhibit cell invasion and migration abilities in vitro using the Transwell and scratch healing assays in BGC-823, SGC-7901, MGC-803 as well as AGS cells. Experiments in a nude mouse model revealed that miR-31 suppressed tumorigenicity in vivo. The luciferase activity assay and western blotting indicated that RhoA was the potential target of miR-31 in GC cells. Collectively, our results provide important evidence that the downregulation of miR-31 inhibited the invasion and migration abilities of GC cells through direct targeting of the tumor
\end{abstract}

Correspondence to: Professor Benyan $\mathrm{Wu}$, Department of Gastroenterology, Division of Southern Building, Chinese PLA General Hospital, 28 Fuxing Road, Beijing 100853, P.R. China E-mail: benyan_wu@163.com

Key words: microRNA, gastric cancer, miR-31, RhoA metastasis-associated gene, RhoA. These findings suggest that miR-31 may be a promising therapeutic candidate in the treatment of GC metastasis.

\section{Introduction}

Gastric cancer (GC) is the fourth most common cancer and the second leading cause of cancer-related deaths worldwide (1). Widespread metastasis is a major factor accounting for the poor prognosis and outcome of GC. Metastasis is considered a complex, multi-step process whereby cancer cells migrate from the primary location to a distant organ (2). This process initiates when primary tumor cells invade their surrounding tissues, followed by tumor cells entering into the blood system (intravasation), translocating through the vasculature, penetrating from the blood stream (extravasation) into the adjacent parenchyma tissue, forming micrometastases and finally proliferating to become secondary gross tumors (3). Many molecular regulators involved in this process have been identified, among which microRNAs (miRNAs) often plays vital roles (4).

miRNAs are small non-coding RNA molecules and play important roles in regulating the expression of various genes by targeting mRNA through translational suppression or cleavage (5). Numerous studies have indicated that these short RNAs (usually 19-25 nucleotides in length) are involved in various biological processes including cell differentiation, proliferation, apoptosis, stress resistance, fat metabolism, tumorigenesis, as well as tumor metastasis (6-8). Numerous studies have revealed that miR-31 expression was specifically attenuated in metastatic breast cancer cells and could inhibit breast cancer metastasis by targeting multiple genes (9). Furthermore, Wang et al found that miR-31 may play an important role in colon cancer metastasis (10). We previously revealed that miR-31 was significantly downregulated in GC tissues through microarray analysis. However, its role in the metastasis of GC has remained largely unknown (11).

In the present study, we investigated the role of miR-31 in the metastasis of GC as well as its underlying mechanisms. We examined the expression of miR-31 in GC and studied its role in GC metastasis by both in vitro and in vivo analysis. We further examined the association of miR-31 expression with lymph node metastasis and found that low expression of miR-31 was associated with lymph node metastasis, poor 
pN stage and invasion into lymphatic vessels. Furthermore, we used bioinformatics analysis and the luciferase reporter assay to identify the potential target of miR-31. The present study, to the best of our knowledge, evaluated for the first time the role of miR-31 in the metastasis of GC using detailed data.

\section{Materials and methods}

Human tissue samples. Seventy-eight pairs of human GC and non-tumor adjacent tissues were obtained from patients who underwent surgical resection at the Chinese PLA General Hospital between 2012 and 2014. All of the samples were clinically and pathologically determined to be correctly labeled and frozen in liquid nitrogen and stored at $-80^{\circ} \mathrm{C}$. No systemic or local treatment was given to these patients before the surgery. The histological grade of the tumor was evaluated based on the World Health Organization (WHO) criteria and patients were staged according to tumor-node-metastasis (TNM) staging of the International Union Against Cancer (UICC)/American Joint Committee on Cancer (AJCC) System (2002). The present study was approved by the Research Ethics Committee of the Chinese PLA General Hospital. Informed consent was obtained from all the patients who provided samples.

Cell culture. The human GC cell lines BGC-823, MGC-803, SGC-7901 and AGS, as well as the normal gastric epithelium cell line GES-1 were obtained from the Chinese Academy of Sciences (Beijing, China) and were all maintained in our own laboratory and cultured in RPMI-1640 medium or Dulbecco's modified Eagle's medium (DMEM) (both from Invitrogen, Carlsbad, CA, USA) supplemented with $10 \%$ fetal bovine serum (FBS; Gibco, Grand Island, NY, USA) in a humidified $5 \%$ carbon dioxide incubator at $37^{\circ} \mathrm{C}$.

RNA extraction and real-time polymerase chain reaction $(R T-P C R)$. Total RNA was extracted from tissues or cultured cells using the mirVana miRNA Isolation kit (Ambion, Foster City, CA, USA) based on the manufacturer's instructions. The poly(A) tail was added to the RNA using the Poly(A) Tailing kit (Ambion). Complementary DNA (cDNA) was synthesized using the TaqMan Reverse Transcription kit (Applied Biosystems, Foster City, CA, USA). Real-time PCR analyses were performed using the TaqMan Micro-RNA Assay kit (Applied Biosystems). miRNAs expression was calculated relative to U6 small nuclear RNA. Changes in the expression were calculated by the $\Delta \Delta \mathrm{Ct}$ method (12). The relative expression ratio of miR-31 was expressed as the fold change normalized to the endogenous reference (U6snRNA), and also relative to the non-tumorous controls (adjacent non-tumor tissues and the GES-1 cell line). Therefore, a relative expression ratio of $<1.0$ was considered to be a low expression level, and a ratio $>1.0$ was considered to be a high expression level. The miRNA primer was purchased from Ambion. The PCR procedure and data analysis were performed using an iCycler (Bio-Rad, Hercules, CA, USA). Each sample was assessed in triplicate. The miR-31 primer sequence was as follows: 5'-AGGCAAGATGCTGGCATAGCT-3'; and the U6-RNA primer sequence was, 5'-TGACACGCAAATTCGT GAAG-3'. All protocols were performed according to the manufacturer's instructions.
RNA oligoribonucleotides and cell transfection. RNA oligoribonucleotides and their corresponding normal controls (NCs) were purchased from RiboBio Co., Ltd. (Guangzhou, China). The cell lines were cultured to $50 \%$ confluence after being transferred into 6-well plates and were then transfected with a final concentration of $100 \mathrm{nM}$ of RNA mimics or $200 \mathrm{nM}$ of inhibitor and their corresponding NCs using Lipofectamine 2000 (Invitrogen) based on the manufacturer's instructions. The cells were harvested for further experiments at $48 \mathrm{~h}$ post-transfection.

In vitro migration/invasion assay. The migratory and invasive abilities of the cell lines were detected using Transwell assay. The Transwells ( $8-\mu \mathrm{m}$ pore size; Corning Costar Corp., Corning, NY, USA) were placed into new 24-well plates. For the Transwell migration assay, $2.5 \times 10^{4}$ cells were plated in the top chamber lined with a non-coated membrane. For the invasion assay, chamber inserts were coated with $35 \mu$ of Matrigel (1:4 dilution) to form the basement membrane and were incubated for $4 \mathrm{~h}$ at $37^{\circ} \mathrm{C}$. Furthermore, $5 \times 10^{4}$ cells were plated in the top chamber. In both assays, the cells were suspended in medium without serum in the lower chamber which was used as a chemoattractant. After incubation at $37^{\circ} \mathrm{C}$ with $5 \% \mathrm{CO}_{2}$ in an incubator, cotton swabs were used to wipe off the upper layer of the Matrigel. After being fixed with 95\% absolute alcohol and stained with 4'6-diamidino-2-phenylindole (DAPI), the number of cells capable of migrating to the lower chamber was thus calculated by inverted microscopy (Olympus Corp., Tokyo, Japan) at a magnification of x200 in over 10 random fields for each well of the plate, and therefore the mean of the number of cells in each visual field represented the invasion ability of the cells. Each experiment was conducted in triplicate.

Scratch wound-healing assays. The cells were cultured into 6 -well plates at a density of $3 \times 10^{5}$ cells/well. After being cultured for $24 \mathrm{~h}$ at $37^{\circ} \mathrm{C}$ in an incubator with $5 \% \mathrm{CO}_{2}$, the cells were then transfected with miR-31 mimics and NC. A straightline scratch was made on the bottom of the cell culture plate using a sterile $200-\mu$ l yellow pipette tip $5 \mathrm{~h}$ post-transfection. Fresh and complete media were added, and the wound healing ability was observed for $24 \mathrm{~h}$. Images were obtained every $8 \mathrm{~h}$.

In vivo metastasis assay. After transfection with the miR-31 mimics or stable NC using trypsin, SGC-7901 cell lines were harvested from tissue culture flasks and were washed three times with phosphate-buffered saline. Next, $5 \times 10^{5}$ cells/1 were suspended in $0.2 \mathrm{ml}$ of serum-free RPMI-1640 medium and were injected into the lateral tail vein of each mouse (six in each group, female BALB/c, 6-8 weeks of age). The mice were sacrificed five weeks post-injection. The number of visible tumor lesions on the lung surface was counted. The lung tissues were then cut into serial sections, fixed with phosphate-buffered neutral formalin, stained with hematoxylin and eosin and then examined histologically. Nude mice were manipulated and cared for according to the NIH Animal Care and Committee guidelines of the Experimental Animal Center of the Chinese PLA General Hospital.

Bioinformatics analysis. The miRNA target predictions were obtained from PicTar (http://pictar.mdc-berlin.de/), 


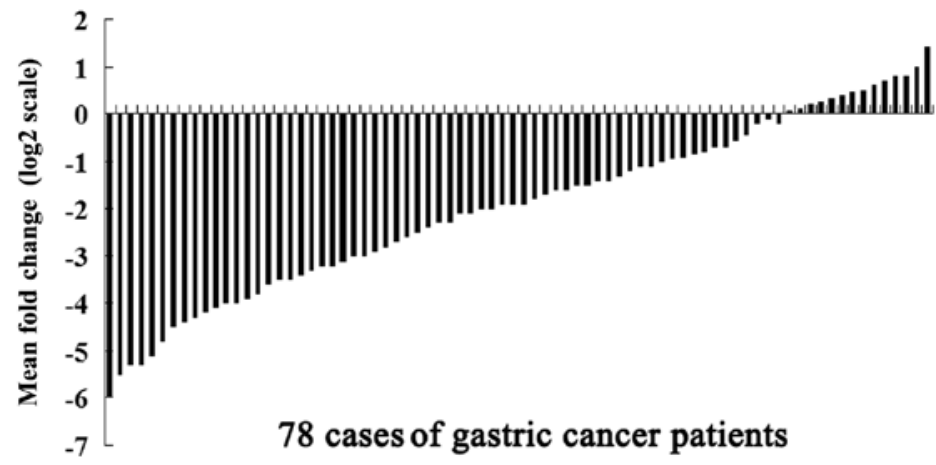

Figure 1. Relative expression of miR-31 in 78 patients with gastric cancer (GC). The quantification of microRNAs (miRNAs) was assessed by quantitative reverse transcription real-time polymerase chain reaction (qRT-PCR). The data are presented as $\log 2$ of the mean fold change in GC relative to non-tumor adjacent tissues. The cases below the line $(\log 2=-1)$ revealed a $>50 \%$ decrease in the miR-31 expression level.

TargetScan 7.0 (http://www.targetscan.org/vert-70/) and miRDB (http://mirdb.org/miRDB). The overlapping targets were further studied using the Expression Analysis Systematic Explorer (EASE) based on the Gene Ontology database.

Vector construction and luciferase reporter assay. Luciferase reporters were constructed based on the firefly luciferase-expressing vector pGL3-control (Promega, Madison, WI, USA). To construct the pGL3-RhoA-3' untranslated region (3'UTR), a partial 3'UTR of the RhoA segment of human RhoA mRNA (GenBank accession no. NM_001664) containing the putative miR-31 binding sites was thus amplified and cloned into the pGL3-control vector. The following primers were used for the amplification of RhoA: forward, 5'-GGCTGCCATCCGGAAGAAA-3'; and reverse, 5'-CACA AGACAAGGCACCCAGA-3'. In addition, we constructed a luciferase reporter that had DNA segments with scrambled target sites to miR-31 as a positive control.

SGC-7901 cells were transfected using Lipofectamine 2000 in 24-well plates based on the manufacturer's instructions, with $0.8 \mu \mathrm{g}$ of the firefly luciferase reporter vector and $0.08 \mu \mathrm{g}$ of the control vector containing Renilla luciferase, pRL-TK (Promega). miR-31 (40 nM) or NC was used for each well. Firefly and Renilla luciferase activities were detected using a Dual-Luciferase Reporter Assay (Promega) $24 \mathrm{~h}$ post-transfection using the Centro LB 960 system (Berthold Technologies, Bad Wildbad, Germany).

Western blotting. Total protein was extracted from the cultured cells using the total protein extraction kit based on the manufacturer's instructions (KeyGen Biotech Co., Ltd., Nanjing, China). Proteins were separated by $8 \%$ SDS polyacrylamide gels and were then transferred to polyvinylidene difluoride membranes (Millipore, Billerica, MA, USA) electrophoretically. The membranes were blocked with $5 \%$ non-fat milk in TBS with $0.05 \%$ Tween-20 (TBST) at $37^{\circ} \mathrm{C}$ for $2 \mathrm{~h}$. Antibodies targeting against RhoA (1:200; Santa Cruz Biotechnology, Santa Cruz, CA, USA) and $\beta$-actin (1:4,000; Sigma-Aldrich, St. Louis, MO, USA) were used. The proteins were visualized using an enhanced chemiluminescence (ECL) kit (Pierce, Rockford, IL, USA) and MF-Chemi BIS 3.2 Pro (Micro Photonics, Allentown, PA, USA) with GelCapture software (DNR Bio-Imaging Systems, Ltd., Jerusalem,
Israel). The intensity of protein fragments was quantified by FluorChem 2.01 software (Alpha Innotech, San Leandro, CA, USA).

Statistical analysis. Data are presented as the means \pm standard deviation (SD) based on at least three separate experiments. Statistical analysis was performed using Student's t-test, a nonparametric test (Mann-Whitney U test between two groups and Kruskal-Wallis test for three or more groups). The correlations between the expression of miR-31 and RhoA protein were calculated by Chi-squared test and Spearman's rank correlation. Differences were considered to be statistically significant for a P-value $<0.05$. Statistical analysis was performed using SPSS 16 (SPSS, Inc., Chicago, IL, USA).

\section{Results}

miR-31 expression and its correlation with the clinicopathological characteristics of GC. miR-31 was detected in all 78 pairs of GC tissues, and their matched non-tumor adjacent tissues using quantitative reverse transcription real-time PCR (qRT-PCR) analysis. Among the 78 patients with GC, $53(67.95 \%)$ cases showed a $>50 \%$ decrease in the miR-31 expression level relative to their matched adjacent non-tumor tissues (Fig. 1). We then studied the correlation between miR-31 expression and the clinicopathological characteristics of GC. The Mann-Whitney U test revealed that lower expression levels of miR-31 were associated with higher lymph node metastasis, poorer $\mathrm{pT}$ and $\mathrm{pN}$ stage (Table I)

To further validate the role of miR-31 in GC cell metastasis, we examined the mRNA expression levels of miR-31 in four human GC cell lines: BGC-823, SGC-7901, MGC-803 and AGS. As shown in Fig. 2, the expression of miR-31 was lower in the BGC-823 and SGC-7901 cells, which had a relatively high metastatic potential, while miR-31 was highly expressed in the MGC-803 and AGS cells with a relatively low metastatic potential. These results revealed that the expression of miR-31 was negatively correlated with GC metastasis and may play an important role in GC metastasis.

miR-31 inhibits the invasion and migration of GC cells in vitro. The significant low expression of the miR-31 in GC cell lines with high metastatic potential prompted us to further 


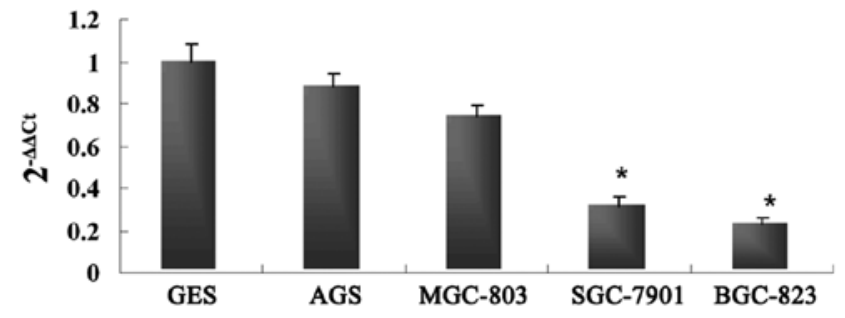

Relative expression of miR-31 in gastric cancer cell lines

Figure 2. Relative expression of miR-31 in four gastric cancer cell lines (MGC-803, SGC-7901, BGC-823 and AGS). The quantification of miR-31 was assessed by qRT-PCR. The data are presented in GC cell lines relative to GES-1 (which is a normal gastric epithelial cell line that was chosen as a control). The results represent the means of the values. The bars indicate the SD. "Statistically significant difference between groups $(\mathrm{P}<0.01)$.

Table I. Expression levels of miR-31 according to the clinicopathological features of patients with gastric cancer.

\begin{tabular}{|c|c|c|c|}
\hline & $\mathrm{N}$ & $\operatorname{miR}-31^{a}$ & P-value \\
\hline Age (years) & & & 0.373 \\
\hline$\leq 50$ & 30 & $0.41(0.08-0.79)$ & \\
\hline$>50$ & 48 & $0.45(0.14-1.20)$ & \\
\hline Sex & & & 0.500 \\
\hline Male & 51 & $0.43(0.10-0.89)$ & \\
\hline Female & 27 & $0.39(0.09-1.21)$ & \\
\hline Tumor size $(\mathrm{cm})$ & & & 0.621 \\
\hline$\leq 3.5$ & 35 & $0.44(0.12-1.25)$ & \\
\hline$>3.5$ & 43 & $0.42(0.15-1.19)$ & \\
\hline Histological grade & & & 0.500 \\
\hline Well & 25 & $0.41(0.15-0.98)$ & \\
\hline Moderate and poor & 53 & $0.44(0.06-1.34)$ & \\
\hline pT stage & & & $0.01^{\mathrm{a}}$ \\
\hline $\mathrm{T} 1+\mathrm{T} 2$ & 36 & $0.53(0.18-1.07)$ & \\
\hline $\mathrm{T} 3+\mathrm{T} 4$ & 42 & $0.37(0.05-0.96)$ & \\
\hline pN stage & & & $0.03^{\mathrm{a}}$ \\
\hline N0 & 24 & $0.73(0.19-1.38)$ & \\
\hline N1 & 20 & $0.36(0.11-0.94)$ & \\
\hline N2 & 19 & $0.29(0.05-0.66)$ & \\
\hline N3 & 15 & $0.24(0.03-0.71)$ & \\
\hline $\begin{array}{l}\text { Invasion into } \\
\text { lymphatic vessels }\end{array}$ & & & $0.000^{\mathrm{a}}$ \\
\hline Negative & 33 & $0.63(0.19-1.34)$ & \\
\hline Positive & 45 & $0.25(0.07-0.67)$ & \\
\hline
\end{tabular}

${ }^{\mathrm{a}}$ Median relative expression of miR-31, with means \pm SD. The mean fold changes in miR-31 expression were calculated using the $2^{-\Delta \Delta C t}$ method. ${ }^{*}$ Statistically significant at $\mathrm{P}<0.05$.

explore the potential biological significance of miR-31 in GC metastasis. We investigated the effects of miR-31 expression on the metastatic abilities of GC cells with different metastatic potential in vitro. An inhibitor and an $\mathrm{NC}$ oligonucleotide (MGC-803-inhibitor and MGC-803-NC, respectively) were thus, introduced into MGC-803 cells to perform metastasis assays in vitro. In addition, miR-31 mimics and an NC oligonucleotide (BGC-823-miR-31-mimics and BGC-823-NC) were constructed and introduced into BGC- 823 cells. The results revealed that the depletion of miR-31 significantly enhanced the invasion and migration abilities of MGC-803 cells as determined by Transwell assay (Fig. 3A). Conversely, the increased expression of miR-31 significantly inhibited the invasion and migration abilities of BGC-823 cells (Fig. 3B).

Wound scratch assay results revealed that the cell migration distances in the BGC-823-miR-31-mimics and BGC-823-NC cells were $537 \pm 22$ and $210 \pm 14 \mu \mathrm{m}$, respectively at $24 \mathrm{~h}$ after the wound scratch. There was a significant difference in the cell migration distance between the BGC-823-miR-31-mimics and the BGC-823-NC. In addition, the results indicated that the miR-31-mimics significantly inhibited the migration ability of the BGC-823 cells while the miR-131 inhibitor promoted the migration ability of the SGC-7901 cells.

miR-31 suppresses GC metastasis in a nude mouse xenograft model. To further confirm the aforementioned findings, we performed an in vivo study using a nude mouse xenograft model. SGC-7901 cells transfected with the miR-31 mimics or NC were injected into the lateral tail vein of nude mice, and the mice were sacrificed five weeks after inoculation. The number of lung metastatic lesions were markedly decreased in the nude mice injected with the miR-31 mimic-transfected cells compared with the negative control-injected ones (Fig. 5). These findings provide strong evidence that miR-31 also inhibited tumor metastasis in vivo.

RhoA may be a functional target of miR-31 in the process of GC metastasis. To investigate how the low expression of miR-31 contributes to the enhanced metastatic ability of GC, we explored potential regulatory targets of miR-31 using the combination of prediction tools, including PicTar, TargetScan and miRBase target. Although hundreds of different targets could be predicted, the genes involved in the migration or invasion process may be the real relevant targets related to the biological functions of miR-31. We next performed functional classification of the predicted targets using the Database for Annotation, Visualization and Integrated Discovery (DAVID) program (http://david.abcc.ncifcrf.gov/). Among these genes, RhoA attracted our attention and may possibly contribute to the metastasis of GC.

To confirm that RhoA was the direct target of miR-31, we constructed the luciferase reporter pGL3-RhoA-3'UTR. The scrambled target site pGL3-RhoA-MUT was also constructed as a negative control. All reporters were transfected into SGC-7901 cells. The luciferase activity of the pGL3-RhoA-3'UTR was significantly suppressed in the SGC-7901-miR-31-mimic cells compared with that in SGC-7901-NC cells when normalized to the control vector pRL-TK, containing Renilla luciferase. (Fig. 6). However, no significant difference in the relative luciferase activity of the pGL3-RhoA-MUT reporters was observed in the SGC-7901miR-31-mimics compared with that in the SGC-7901-NC. These results revealed that RhoA proteins were negatively and directly regulated by miR-31 in GC cells, and that RhoA may serve as a target of miR-31. 
A

\section{$\mathbf{A}$ \\ Invasion}

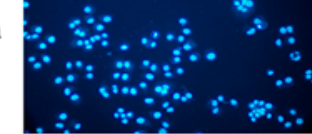

MGC-803-NC

$\mathbf{B}$

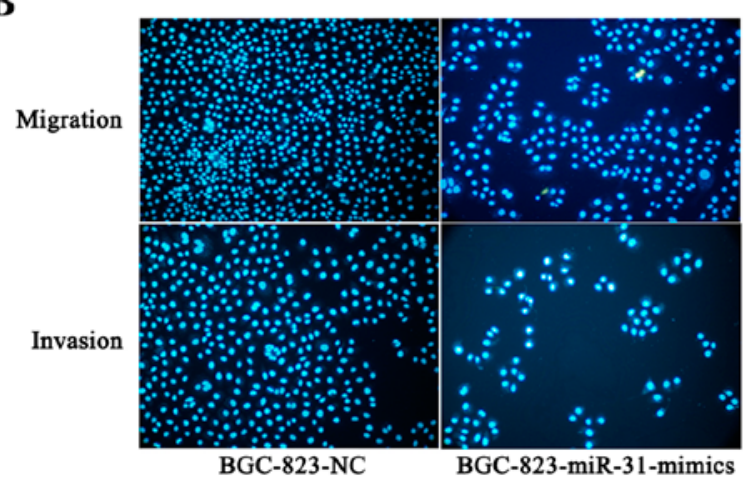

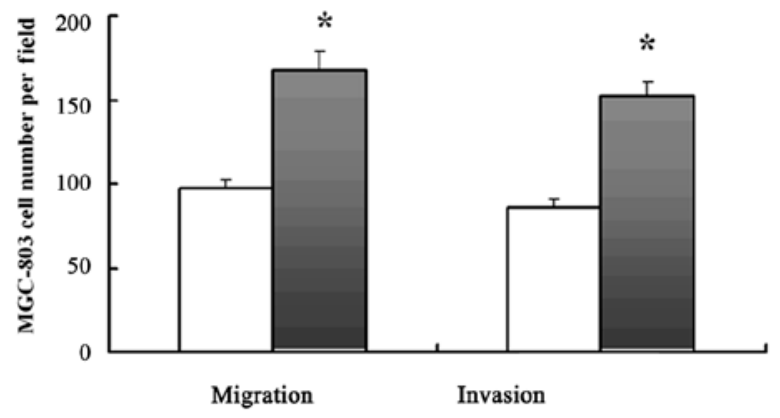

口MGC-803-NC EMGC-803-inhibitor

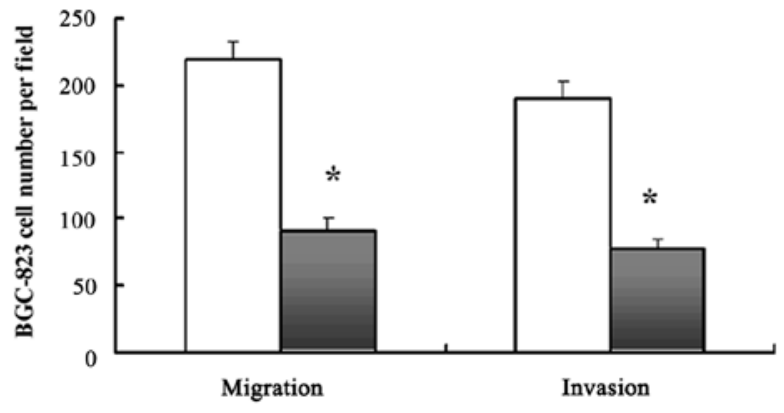

口BGC-823-NC घBGC-823-miR-31-mimics

Figure 3. Effect of miR-31 on tumor cell invasion and the metastasis of MGC-803 and BGC-823 cells. (A) Invasion and migration assay. Representative fields of migration (upper images) or invasive (lower images) cells on the membrane (left) (magnification of x200). Average invasive or migration cell number/field (right). The migration or invasive cell number of MGC-803 cells transfected with miR-31-inhibitors was markedly increased compared with the cells transfected with the negative control; ${ }^{*} \mathrm{P}<0.05$, Student's t-test, $\mathrm{n}=10$. (B) The migration or invasive cell number of BGC-823 cells transfected with miR-31-mimics was markedly decreased compared with those transfected with the negative control. ${ }^{*} \mathrm{P}<0.05$, Student's $\mathrm{t}$-test, $\mathrm{n}=10$.

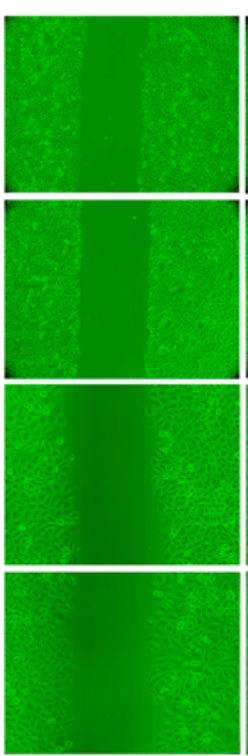

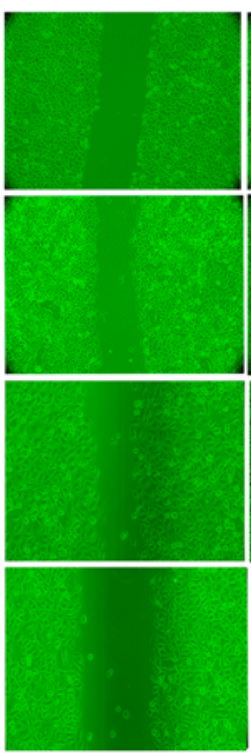

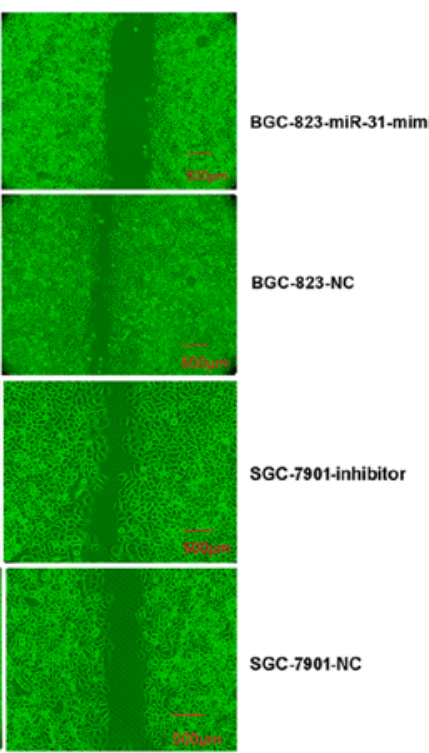

$24 \mathrm{~h}$

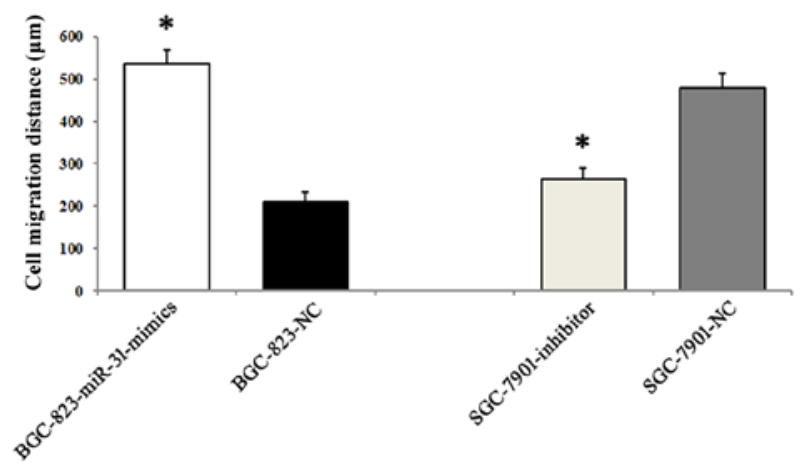

Figure 4. Effect of miR-31 on gastric cancer (GC) cell migration using the wound-healing assay. Cells were transfected 5 h before the wound-healing assay. The cell migration distances were assessed every $8 \mathrm{~h}$ after the 'wound'. Scale bar, $500 \mu \mathrm{m}$. ${ }^{*} \mathrm{P}<0.05$.

To furtherconfirm these findings, weexamined RhoAprotein levels in both miR-31-transfected and anti-miR-31-transfected cells, as well as in their corresponding $\mathrm{NC}$ and parental SGC-7901 cells, using western blotting and real-time qRT-PCR analysis. We observed a significant decrease in the level of the endogenous RhoA proteins in SGC-7901-miR-31-mimic cells than that in the SGC-7901-NC cells when normalized to the endogenous reference $\beta$-actin protein. Overexpression of RhoA proteins was also observed in the anti-miR-31-transfected SGC-7901 cells compared with that in the anti-NC-transfected and parental SGC-7901 cells (Fig. 7). These results demonstrated that miR-31 may target and regulate RhoA in GC. 


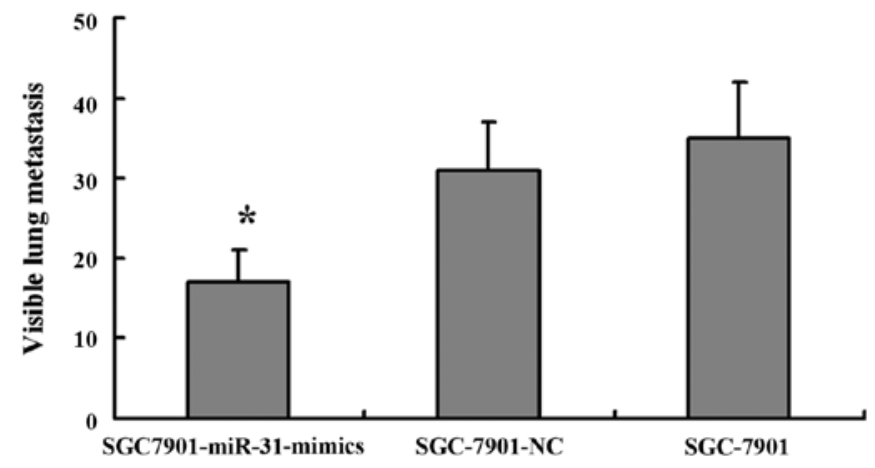

Figure 5. miR-31 suppresses GC metastasis in vivo. The data are displayed graphically with the number of lung metastases from each group of mice. Results represent the mean values. The bars indicate the SD. *Statistically significant difference between groups $(\mathrm{P}<0.05)$. GC, gastric cancer.

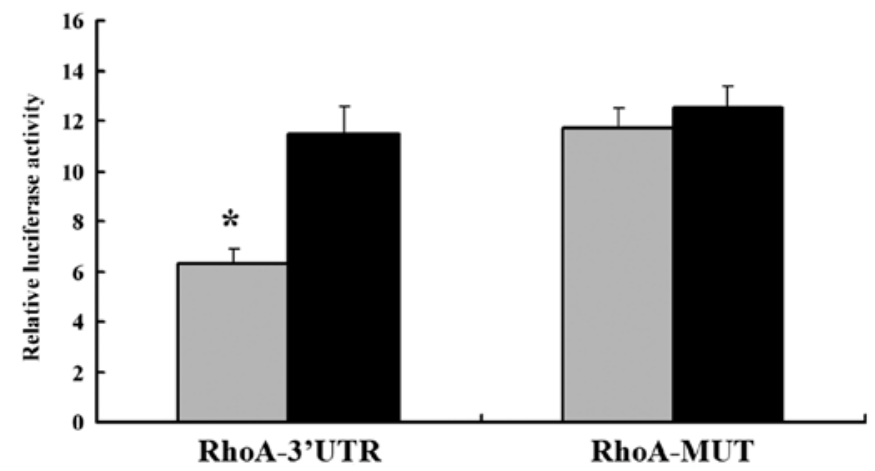

Figure 6. miR-31 directly targets RhoA. Analysis of the luciferase activity of RhoA and RhoA-MUT with miR-31 mimics or normal controls (NCs) in the SGC-7901 cells. *Statistically significant difference between groups $(\mathrm{P}<0.05)$.
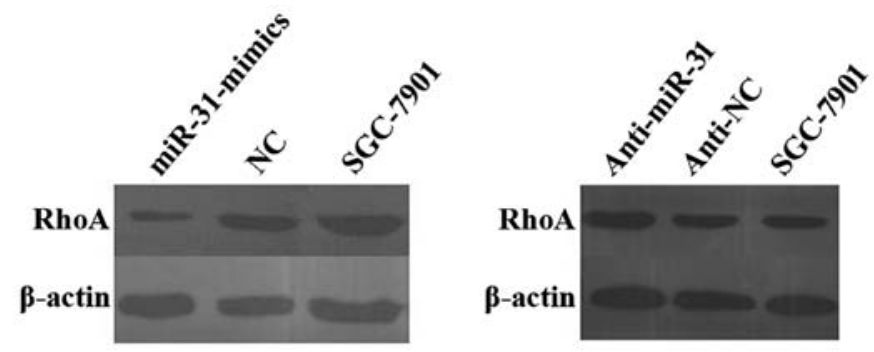

Figure 7. RhoA proteins are potential targets of miR-31 in gastric cancer cells Protein expression analysis for RhoA in miR-31-transfected, NC-transfected and parental SGC-7901 cells as determined by western blotting. A greater decrease in the level of the endogenous RhoA proteins was demonstrated in the miR-31-transfected SGC-7901 cells compared with the NC-transfected SGC-7901 cells normalized to the endogenous reference $\beta$-actin protein. The level of the endogenous RhoA proteins was increased in the anti-miR31-transfected SGC-7901 cells compared with that in the anti-NC-transfected SGC-7901 cells normalized to the endogenous reference $\beta$-actin protein.

\section{Discussion}

The microRNA-31 (miR-31) gene is located at 9p21.3. Numerous studies have shown that miR-31 has different expression patterns in different types of cancer, for example, it was downregulated in urothelial carcinoma of the bladder (13), breast (9) and serous ovarian cancer (14), while it was upregulated in colorectal cancer (CRC) $(15,16)$, head and neck squamous cell carcinoma (HNSCC) (17), hepatocellular carcinoma (18) and lung cancer (19). Although numerous studies have investigated the different expression levels of miR-31 in different types of cancer, the function of miR-31 still remained unclear.

In the present study, we demonstrated that miR-31 expression was markedly downregulated in both GC tissues and cell lines, suggesting that the low expression of miR-31 may be associated with GC development. Furthermore, we demonstrated that the low expression of miR-31 was significantly associated with a higher rate of lymph node metastasis, poorer $\mathrm{pT}$ and $\mathrm{pN}$ stage, and invasion into lymphatic vessels. Lymph node metastasis, $\mathrm{pT}$ and $\mathrm{pN}$ stage were independent prognostic factors for the overall survival rates of GC patients, and lymphatic vessel invasion was identified as an independent prognostic factor predicting lymph node metastasis. GC patients with lymph node metastasis, poor pT and pN stage and invasion into lymphatic vessels tended to have a lower survival rate $(20,21)$. This suggested that the expression of miR-31 may act as an independent prognostic factor for the overall survival rates of $\mathrm{GC}$ patients.

Recent studies revealed that miR-31 expression was specifically attenuated in metastatic breast cancer cell lines, and miR-31 could inhibit breast cancer metastasis by targeting multiple genes (9). In the present study, we found that overexpression of miR-31 suppressed GC cell invasion and metastatic abilities. These results were consistent with a previous study, which revealed that blockade of miR-31 expression significantly decreased the invasion and migration abilities of the HNSCC cell line (17). The development of GC metastasis is characterized by multiple genetic alterations. Previous studies have investigated the effects of specific miRNAs on the pattern of GC metastasis. Zheng et al found that miRNA-145 inhibited the metastasis and angiogenesis abilities of GC cells by targeting the 3'UTR of Ets1 (22). The expression of let-7f was decreased in gastric tumors compared with that in normal gastric tissue and inhibited tumor metastasis by targeting MYH9 (23). Tie et al found that decreased miR-218 expression was associated with advanced clinical stage lymph node metastasis and poor prognosis in GC patients, and that the overexpression of miR-218 in metastatic cells inhibited migration, invasion, and metastasis formation as shown both by in vitro and in vivo experiments (24). Although numerous studies have investigated the role of miRNA in GC metastasis, the underlying mechanisms remain unclear. Notably, to the best of our knowledge, few studies have investigated whether GC metastasis is regulated by miRNA-31.

To examine the molecular mechanism by which miR-31 functioned as a metastasis suppressor in GC, we used the luciferase reporter assay and western blotting to confirm that RhoA was a potential target of miR-31 in GC cells. RhoA, with a molecular mass of $21 \mathrm{kDa}$, is the most widely studied member of the Rho GTPase family, and belongs to the Ras superfamily of small $\mathrm{G}$ proteins. The Rho GTPase family consists of at least 11 members sharing $>50 \%$ sequence identity, with one of the most well known members being RhoA. RhoA acts as a molecular switch in cells, regulating signal transduction from cell surface receptors to intracellular target molecules, and is involved in various biological process, including cell morphology (25), motility (26), 
cytokinesis $(27,28)$, smooth muscle contraction $(29,30)$ and tumor progression $(31,32)$.

In conclusion, the present study demonstrated that miR-31 could suppress the metastasis of GC by directly binding to the 3'UTR of RhoA. Although there is still a lot to learn concerning the role of miR-31 in GC tumorigenesis, miR-31 may serve as a promising potential target for GC treatment.

\section{Acknowledgements}

The present study was supported by the National Ministry of Technology Foundation of China (2009BAI86B05). The authors would like to thank Professor Yao He for his technical assistance.

\section{References}

1. Compare D, Rocco A and Nardone G: Risk factors in gastric cancer. Eur Rev Med Pharmacol Sci 14: 302-308, 2010.

2. Fidler IJ: The pathogenesis of cancer metastasis: The 'seed and soil' hypothesis revisited. Nat Rev Cancer 3: 453-458, 2003.

3. Gupta GP and Massagué J: Cancer metastasis: Building a framework. Cell 127: 679-695, 2006.

4. Ma L and Weinberg RA: Micromanagers of malignancy: Role of microRNAs in regulating metastasis. Trends Genet 24: 448-456, 2008.

5. Zamore PD and Haley B: Ribo-gnome: The big world of small RNAs. Science 309: 1519-1524, 2005.

6. Kloosterman WP and Plasterk RH: The diverse functions of microRNAs in animal development and disease. Dev Cell 11: 441-450, 2006

7. Esquela-Kerscher A and Slack FJ: Oncomirs - microRNAs with a role in cancer. Nat Rev Cancer 6: 259-269, 2006.

8. Lu J, Getz G, Miska EA, Alvarez-Saavedra E, Lamb J, Peck D, Sweet-Cordero A, Ebert BL, Mak RH, Ferrando AA, et al: MicroRNA expression profiles classify human cancers. Nature 435: 834-838, 2005.

9. Valastyan S, Reinhardt F, Benaich N, Calogrias D, Szász AM, Wang ZC, Brock JE, Richardson AL and Weinberg RA: A pleiotropically acting microRNA, miR-31, inhibits breast cancer metastasis. Cell 137: 1032-1046, 2009.

10. Wang CJ, Stratmann J, Zhou ZG and Sun XF: Suppression of microRNA-31 increases sensitivity to 5-FU at an early stage, and affects cell migration and invasion in HCT-116 colon cancer cells. BMC Cancer 10: 616, 2010.

11. Zhang WH, Gui JH, Wang CZ, Chang Q, Xu SP, Cai CH, Li YN, Tian YP, Yan L and Wu B: The identification of miR-375 as a potential biomarker in distal gastric adenocarcinoma. Oncol Res 20: 139-147, 2012.

12. Livak KJ and Schmittgen TD: Analysis of relative gene expression data using real-time quantitative PCR and the $2^{-\Delta \Delta C T}$ method. Methods 25: 402-408, 2001.

13. Schaefer A, Jung M, Mollenkopf HJ, Wagner I, Stephan C, Jentzmik F, Miller K, Lein M, Kristiansen G and Jung K: Diagnostic and prognostic implications of microRNA profiling in prostate carcinoma. Int J Cancer 126: 1166-1176, 2010.

14. Creighton CJ, Fountain MD, Yu Z, Nagaraja AK, Zhu H, Khan M, Olokpa E, Zariff A, Gunaratne PH, Matzuk MM, et al: Molecular profiling uncovers a $\mathrm{p} 53$-associated role for microRNA-31 in inhibiting the proliferation of serous ovarian carcinomas and other cancers. Cancer Res 70: 1906-1915, 2010.

15. Wang CJ, Zhou ZG, Wang L, Yang L, Zhou B, Gu J, Chen HY and Sun XF: Clinicopathological significance of microRNA-31, -143 and -145 expression in colorectal cancer. Dis Markers 26: 27-34, 2009.
16. Motoyama K, Inoue H, Takatsuno Y, Tanaka F, Mimori K, Uetake H, Sugihara K and Mori M: Over- and under-expressed microRNAs in human colorectal cancer. Int J Oncol 34: 1069-1075, 2009.

17. Liu CJ, Tsai MM, Hung PS, Kao SY, Liu TY, Wu KJ, Chiou SH, Lin SC and Chang KW: $m i R-31$ ablates expression of the HIF regulatory factor FIH to activate the HIF pathway in head and neck carcinoma. Cancer Res 70: 1635-1644, 2010.

18. Wong QW, Lung RW, Law PT, Lai PB, Chan KY, To KF and Wong N: MicroRNA-223 is commonly repressed in hepatocellular carcinoma and potentiates expression of Stathmin1. Gastroenterology 135: 257-269, 2008.

19. Liu X, Sempere LF, Ouyang H, Memoli VA, Andrew AS, Luo Y, Demidenko E, Korc M, Shi W, Preis M, et al: MicroRNA-31 functions as an oncogenic microRNA in mouse and human lung cancer cells by repressing specific tumor suppressors. J Clin Invest 120: 1298-1309, 2010.

20. Sun Z, Li DM, Wang ZN, Huang BJ, Xu Y, Li K and Xu HM: Prognostic significance of microscopic positive margins for gastric cancer patients with potentially curative resection. Ann Surg Oncol 16: 3028-3037, 2009.

21. Jiang CG, Wang ZN, Sun Z, Liu FN, Yu M and Xu HM: Clinicopathologic characteristics and prognosis of gastric cancer invading the subserosa. J Surg Oncol 102: 737-741, 2010.

22. Zheng L, Pu J, Qi T, Qi M, Li D, Xiang X, Huang K and Tong Q: miRNA-145 targets v-ets erythroblastosis virus E26 oncogene homolog 1 to suppress the invasion, metastasis, and angiogenesis of gastric cancer cells. Mol Cancer Res 11: 182-193, 2013.

23. Liang S, He L, Zhao X, Miao Y, Gu Y, Guo C, Xue Z, Dou W, $\mathrm{Hu} \mathrm{F}$, Wu K, et al: MicroRNA let-7f inhibits tumor invasion and metastasis by targeting MYH9 in human gastric cancer. PLoS One 6: e18409, 2011.

24. Tie J, Pan Y, Zhao L, Wu K, Liu J, Sun S, Guo X, Wang B, Gang Y, Zhang Y, et al: MiR-218 inhibits invasion and metastasis of gastric cancer by targeting the Robol receptor. PLoS Genet 6: e1000879, 2010

25. Paterson HF, Self AJ, Garrett MD, Just I, Aktories K and Hall A: Microinjection of recombinant p21rho induces rapid changes in cell morphology. J Cell Biol 111: 1001-1007, 1990.

26. Takaishi K, Kikuchi A, Kuroda S, Kotani K, Sasaki T and Takai Y: Involvement of rho p21 and its inhibitory GDP/GTP exchange protein (rho GDI) in cell motility. Mol Cell Biol 13: 72-79, 1993.

27. Kishi K, Sasaki T, Kuroda S, Itoh T and Takai Y: Regulation of cytoplasmic division of Xenopus embryo by rho p21 and its inhibitory GDP/GTP exchange protein (rho GDI). J Cell Biol 120: 1187-1195, 1993.

28. Jantsch-Plunger V, Gönczy P, Romano A, Schnabel H, Hamill D, Schnabel R, Hyman AA and Glotzer M: CYK-4: A Rho family gtpase activating protein (GAP) required for central spindle formation and cytokinesis. J Cell Biol 149: 1391-1404, 2000.

29. Hirata K, Kikuchi A, Sasaki T, Kuroda S, Kaibuchi K, Matsuura Y, Seki H, Saida K and Takai Y: Involvement of rho p21 in the GTP-enhanced calcium ion sensitivity of smooth muscle contraction. J Biol Chem 267: 8719-8722, 1992.

30. Gong MC, Iizuka K, Nixon G, Browne JP, Hall A, Eccleston JF, Sugai M, Kobayashi S, Somlyo AV and Somlyo AP: Role of guanine nucleotide-binding proteins - ras-family or trimeric proteins or both - in $\mathrm{Ca}^{2+}$ sensitization of smooth muscle. Proc Natl Acad Sci USA 93: 1340-1345, 1996.

31. Perona R, Esteve P, Jiménez B, Ballestero RP, Ramón y Cajal S and Lacal JC: Tumorigenic activity of rho genes from Aplysia californica. Oncogene 8: 1285-1292, 1993.

32. Prendergast GC, Khosravi-Far R, Solski PA, Kurzawa H, Lebowitz PF and Der CJ: Critical role of Rho in cell transformation by oncogenic Ras. Oncogene 10: 2289-2296, 1995. 